\title{
Changes in Age at Diagnosis of Type 2 Diabetes Mellitus in the United States, 1988 to 2000
}

Richelle J. Koopman, MD, MS

Arcb G. Mainous III, PbD

Vanessa A. Diaz, MD, MS

Mark E. Geesey, MS

Department of Family Medicine, Medical University of South Carolina, Charleston, SC
Conflicts of interest: none reported

\section{CORRESPONDING AUTHOR}

Richelle Koopman, MD, MS Department of Family Medicine Medical University of South Carolina 295 Calhoun Street, PO Box 250192 Charleston, SC 29425

koopmanr@musc.edu

\begin{abstract}
PURPOSE The prevalence of diabetes in the United States is increasing. There is also concern that diabetes may be occurring at a greater frequency in youth and in young adults. We describe US population trends in self-reported age at diagnosis of type 2 diabetes mellitus.
\end{abstract}

METHODS We undertook a secondary analysis of data from the National Health and Nutrition Examination Survey (NHANES) 1999-2000 and NHANES III (19881994). Both surveys are stratified, multistage probability samples targeting the civilian, noninstitutionalized US population, which allow calculation of population estimates. We included adults aged 20 years and older. We compared selfreported age at diagnosis of type 2 diabetes between the 2 survey periods.

RESULTS The mean age at diagnosis decreased from 52.0 to 46.0 years $(P<.05)$. Racial and ethnic differences in age at diagnosis found in 1988 to 1994 are no longer found in 1999 to 2000.

CONCLUSIONS The age at diagnosis of type 2 diabetes mellitus has decreased with time. This finding likely represents a combination of changing diagnostic criteria, improved physician recognition of diabetes, and increased public awareness. Younger age at diagnosis may also reflect a true population trend of earlier onset of type 2 diabetes.

Ann Fam Med 2005;3:60-63. DOI: 10.1370/afm.214.

\section{INTRODUCTION}

$\mathrm{T}$ The prevalence of diagnosed diabetes in the United States is increasing, coincident with increases in the prevalence of obesity and sedentary lifestyle..$^{1-3}$ Among adults in the United States, population estimates of the prevalence of diagnosed diabetes increased from $3.4 \%$ in 1976 to 1980 , to $5.1 \%$ in 1988 to 1994 , to $5.9 \%$ in 1999 to $2000{ }^{4-6}$ Rates of diagnosed diabetes for racial and ethnic minorities generally exceeded that of non-Hispanic whites. ${ }^{4 \cdot 6}$

There has been concern that child and adolescent age-groups have also been affected by rising trends in type 2 diabetes mellitus. ${ }^{7.8}$ The prevalence of overweight children and adolescents is increasing in the United States. ${ }^{9,10}$ Although there have not been US population estimates of the prevalence of type 2 diabetes in youth, an increase in type 2 diabetes has been noted in pediatric diabetes referral centers, as well as in Pima Indians. ${ }^{11,12}$ Additionally, there have not been estimates of population trends in age of onset of type 2 diabetes among adults in the United States.

Given the increasing prevalence of diabetes in the United States and the evidence that type 2 diabetes is being diagnosed in younger populations, the onset of type 2 diabetes may be occurring at a much younger age in the US population as a whole. An understanding of this trend will 
help define the magnitude of this health problem. We report the mean self-reported age at diagnosis of type 2 diabetes and make comparisons between the most recently available population data from the National Health and Nutrition Examination Survey (NHANES 1999-2000), and data from the Third National Health and Nutrition Examination Survey (NHANES III) (1988-1994).

\section{METHODS}

\section{Data Sources}

We analyzed data from the NHANES 1999-2000 and the NHANES III. The National Center for Health Statistics (NCHS), Division of Health Examination Statistics, part of the Centers for Disease Control and Prevention (CDC), has conducted the NHANES series of health and nutrition surveys since the early 1960s. The most recent NHANES spans the years 1999 to 2000 and the NHANES III spans the years 1988 to 1994 . Both surveys include a household interview, physician examination, laboratory testing, and other diagnostic testing at mobile examination centers. The NHANES survey designs are stratified, multistage probability samples that target the civilian, noninstitutionalized US population. The sampling design and weighting system of the surveys allow calculation of estimates of the number and percentage of persons in the US population and designated age and race/ethnicity subgroups with selected diseases, despite intentional oversampling of children and minorities. ${ }^{13}$ Although the NHANES III spans 6 years, the NCHS recommends that computation of national estimates from the total NHANES III is the preferred analysis method, because individual phase estimates may be highly variable. ${ }^{13}$

\section{Assessment of Diagnosed Diabetes and Age at Diagnosis}

The NHANES surveys assessed participants for diagnosed diabetes using the question, "Other than during pregnancy, have you ever been told by a doctor or health professional that you have diabetes or sugar diabetes?" We defined participants with diagnosed diabetes as those who answered "yes" to this question about the presence of diagnosed diabetes, which excluded gestational diabetes mellitus. Age at diagnosis for those defined as having diagnosed diabetes was determined by the participant's self-report of their age "when a health professional first told you that you had diabetes or sugar diabetes." Participants with diabetes who did not report their age at diagnosis were excluded ( $\mathrm{n}=27$ for NHANES III $; \mathrm{n}=5$ for NHANES 1999-2000).

\section{Differentiating Type 1 from Type 2 Diabetes Mellitus}

Because we wanted to investigate differences in age at diagnosis of type 2 diabetes mellitus, it was necessary to differentiate type 2 diabetes from type 1 diabetes in NHANES participants. These 2 disease processes are very different and tend to have different ages of onset. NHANES, however, does not specifically categorize participants with regard to type of diabetes and does not measure islet cell autoantibodies. Considering the usual clinical course of type 1 diabetes, we defined anyone with diabetes diagnosed before the age of 30 years who was taking only insulin therapy as having type 1 diabetes mellitus and excluded that person from our sample. This definition is consistent with United Kingdom Prospective Diabetes Study Group (UKPDS) findings that autoantibodies to islet-cell cytoplasm and glutamic acid decarboxylase decline markedly with age. ${ }^{14}$

\section{Stratification by Age and Race/Ethnicity}

We stratified groups by participant self-identified race/ethnicity. Race/ethnicity groups are defined by the NHANES as non-Hispanic white, non-Hispanic black, Mexican American, other, and other Hispanic. For our analyses we combined the Mexican American and other Hispanic groups into a single Hispanic group. We judged the "other" group to be both too heterogeneous and small to make meaningful population estimates, consistent with the NCHS guidelines for analysis of the NHANES III ${ }^{13}$ and this group was excluded from the analysis ( $\mathrm{n}=155$ for NHANES III $\mathrm{n}=274$ for NHANES 1999-2000).

\section{Analysis}

Statistical analyses were carried out using SUDAAN version 8.0.2 (Research Triangle Institute, Research Triangle Park, NC) to account for the complex weighting scheme and clustering of the NHANES surveys and to generate US population estimates from the data. Average age at diagnosis of diabetes is reported for each race/ethnicity group after using appropriate sampling weights from each NHANES survey to arrive at population estimates. We tested for differences in age at diagnosis by race/ethnicity using a series of $t$ tests to compare each race/ethnicity group pair. The Institutional Review Board of the Medical University of South Carolina approved this research.

\section{RESULTS}

The NHANES 1999-2000 contained data on 4,387 persons aged 20 years and older: $47.7 \%$ was male; the average age was 45.0 years; $73.6 \%$ was non-Hispanic white, $11.4 \%$ was non-Hispanic black, and $15.0 \%$ was 
Hispanic. Of these survey participants, we determined that 430 had a diagnosis of type 2 diabetes mellitus. After applying sampling weights, these 430 participants represent 11.6 million persons with type 2 diabetes in the United States in 1999-2000. The NHANES III contained data on 17,010 participants aged 20 years and older: $47.6 \%$ was male; the average age was 44.7 years, $79.1 \%$ was non-Hispanic white, $11.3 \%$ was nonHispanic was black, and $9.5 \%$ was Hispanic. Of these participants, we determined that 1,271 had a diagnosis of type 2 diabetes mellitus. After applying sampling weights, these 1,271 survey participants represent 8.5 million people with type 2 diabetes in the United States in 1988 to 1994.

\section{Age at Diagnosis of Type 2 Diabetes Mellitus} During the NHANES III sample period (1988-1994) the mean age at diagnosis of type 2 diabetes mellitus was 52.0 years (Table 1 ). Whites had diabetes diagnosed at a significantly older age (53.2 years) than blacks (48.5 years, $P<.001$ ) but not Hispanics (50.3 years, $P=.09$ ). Using data from NHANES 1999-2000, the mean age at diagnosis of type 2 diabetes decreased to 46.0 years (Table 1 ). Age at diagnosis did not differ significantly by race/ethnicity $(P=.58$ for whites vs blacks or Hispanics) in the more recent NHANES 1999-2000.

\section{DISCUSSION}

The mean age at diagnosis of type 2 diabetes mellitus in the United States has decreased from 52 years, reported in the years 1988 to 1994 , to 46 years, reported in the years 1999 to 2000 . There are several plausible explanations that might account for this rather dramatic decrease occurring during a course of a decade. This change may represent earlier onset of type 2 diabetes or earlier detection or a combination of these 2 effects. Whether the decrease in age at diagnosis was due to an overall decrease in actual age of onset among American adults or to earlier detection of type 2 diabetes by physicians is unclear and cannot be determined from these data. Decreasing age at diagnosis could also occur through increased public awareness, leading to earlier physician consultation about recognized symptoms of diabetes, which might reflect better education of the population about their risks for diabetes.

Earlier detection by physicians is almost certainly a factor influencing the decreasing age at diagnosis. The diagnostic criteria for diabetes mellitus changed between the time of the NHANES III (1988-1994) and the NHANES 1999-2000 from the criteria of a fasting plasma glucose $\geq 140 \mathrm{mg} / \mathrm{dL}$ or a 2 -hour plasma glucose $\geq 200 \mathrm{mg} / \mathrm{dL}$ to the current American Diabetes Associa-
Table 1. Mean Age at Diagnosis for Persons With Type 2 Diabetes Mellitus From NHANES III (1988-1994) and NHANES 1999-2000

\begin{tabular}{|c|c|c|}
\hline Population & $\begin{array}{c}\text { NHANES III } \\
(1988-1994)\end{array}$ & $\begin{array}{c}\text { NHANES } \\
1999-2000\end{array}$ \\
\hline \multicolumn{3}{|l|}{ Total } \\
\hline Population estimate, $\mathrm{n}$ & $8,475,766$ & $11,633,166$ \\
\hline Mean age at diagnosis, y & 52.04 & 46.01 * \\
\hline Standard error, mean y & 0.54 & 1.27 \\
\hline \multicolumn{3}{|l|}{ White } \\
\hline Population estimate, $\mathrm{n}$ & $6,280,324$ & $6,947,442$ \\
\hline Mean age at diagnosis, y & 53.17 & $46.43^{*}$ \\
\hline Standard error, mean y & 0.66 & 1.90 \\
\hline \multicolumn{3}{|l|}{ Black } \\
\hline Population estimate, $n$ & $1,248,169$ & $2,036,006$ \\
\hline Mean age at diagnosis, y & 48.46 & 45.20 * \\
\hline Standard error, mean y & 0.89 & 1.52 \\
\hline \multicolumn{3}{|l|}{ Hispanic } \\
\hline Population estimate, $n$ & 724,467 & $1,930,094$ \\
\hline Mean age at diagnosis, y & 50.32 & 45.08 * \\
\hline Standard error, mean y & 1.50 & 1.61 \\
\hline \multicolumn{3}{|c|}{$\begin{array}{l}* P<.05 \text { for difference in self-reported mean age at diagnosis of type } 2 \\
\text { diabetes mellitus between NHANES III (1988-1994) and NHANES 1999-2000, } \\
\text { for total population, and for each race/ethnicity stratum. }\end{array}$} \\
\hline
\end{tabular}

tion (ADA) criteria of a fasting plasma glucose of $\geq 126$ $\mathrm{mg} / \mathrm{dL} .{ }^{15,16}$ The institution of yearly updated recommendations for clinical practice and screening from the ADA may play a role in increased detection of diabetes by physicians and other health care clinicians. ${ }^{16,17}$

The United States Preventive Services Task Force (USPSTF) has recommended against mass screening of asymptomatic persons for type 2 diabetes, concluding that the evidence for or against mass screening is inconclusive. ${ }^{18,19}$ The Centers for Disease Control and Prevention (CDC) Diabetes Cost-Effectiveness Study Group concluded that opportunistic screening of all adults older than the age of 25 years would cost $\$ 236,449$ per life-year gained and $\$ 56,649$ per qualityadjusted life-year gained, and it has generally recommended against mass screening for type 2 diabetes in adults. ${ }^{20}$ The ADA does not recommend general population screening, but it does recommend that screening be considered every 3 years for subgroups such as those aged 45 years and older, as well as increased screening based on family history of type 2 diabetes, minority race/ethnicity, and other risk factors. ${ }^{17}$ As suggested by these nationally representative findings of decreasing mean age at diagnosis of type 2 diabetes, these recommendations might need to be reexamined to include clinical screening recommendations for younger age-groups, especially those at high risk. 
A few limitations must be acknowledged. The NHANES did not ask participants to differentiate whether they had type 1 or type 2 diabetes mellitus. For our analysis, we needed to differentiate individuals with these 2 very different disease processes to obtain meaningful data. We believe we have created a construct that allows for the categorization of subjects in a manner that minimizes misclassification bias. As a check on our categorization scheme, we looked at the distribution of $\mathrm{C}$ peptide levels in those that we categorized as having type 2 diabetes in the 1999-2000 data set. We found that among these participants who had the fasting laboratory test performed $(n=156)$, $91.7 \%$ had a measured $C$ peptide $\geq 0.50 \mathrm{nmol} / \mathrm{L}$, consistent with type 2 diabetes. ${ }^{21}$ In addition, both the age at diagnosis and the diagnosis itself are self-reported, which may lead to the question of validity of the diagnosis and correct recall of age at diagnosis. Selfreports of diabetes have been used as the basis of many population-based studies and should not be systematically biased in a way differing from NHANES III to NHANES 1999-2000. ${ }^{2-6}$ Furthermore, memory for age at the time of diagnosis of other health conditions has been shown to be fairly accurate when compared with medical records. ${ }^{22}$

Using the NHANES data set is probably the only existing method to make nationally representative population estimates about age at diagnosis of type 2 diabetes. The observed decrease in the age at diagnosis may reflect population trends in the true age of onset of type 2 diabetes. Alternatively, health care clinicians may be doing a better job at recognizing the subtle symptoms of diabetes. They are also likely to be making earlier diagnoses as a result of more stringent diagnostic criteria. It is further possible that our population may be becoming better educated about these symptoms, leading them to more readily seek appropriate health care. In either case, it is likely that we are detecting type 2 diabetes earlier in the course of the disease, with likely positive health benefits for this population. Identification of factors that can further improve our detection of type 2 diabetes may be a promising area for future research.

To read or post commentaries in response to this article, see it online at http://www.annfammed.org/cgi/content/full/3/1/60.

Key words: Diabetes mellitus/diagnosis; diabetes mellitus/epidemiology; NHANES

Submitted October 23, 2003; submitted, revised, January 30, 2004; accepted February 17, 2004.

Funding support: This research was funded by grants 1 D14 HP 00161 02 and 2 D12-HP-00023-04 from the Health Resources and Services Administration.

\section{References}

1. Flegal KM, Carroll MD, Ogden $\mathrm{CL}$, Johnson $\mathrm{CL}$. Prevalence and trends in obesity among US adults, 1999-2000. JAMA. 2002;288:1723-1727.

2. Mokdad AH, Bowman BA, Ford ES, Vinicor F, Marks JS, Koplan JP. The continuing epidemics of obesity and diabetes in the United States. JAMA. 2001;286:1195-1200.

3. Mokdad AH, Ford ES, Bowman BA, et al. Prevalence of obesity, diabetes, and obesity-related health risk factors, 2001. JAMA. 2003;289:76-79.

4. Harris MI, Goldstein DE, Flegal KM, et al. Prevalence of diabetes, impaired fasting glucose, and impaired glucose tolerance in US adults. Diabetes Care. 1998;518-524.

5. Cowie CC, Rust KF, Byrd-Holt D, et al. Prevalence of diabetes and impaired fasting glucose in adults-United States, 1999-2000. MMWR. 2003;52:833-837.

6. National Center for Health Statistics, Hadden WC, Harris MI. Public Health Service. Washington, US Government Printing Office. Prevalence of diagnosed diabetes, undiagnosed diabetes, and impaired glucose tolerance in adults 20-74 years of age, United States, 1976 80. Vital and Health Statistics, Feb 1987; Series 11, No. 237. DHHS Publication No. (PHS) 87-1687.

7. Rocchini AP. Childhood obesity and a diabetes epidemic. $N$ Engl J Med. 2002;346:854-855.

8. Fagot-Campagna A, Pettitt DJ, Engelgau MM, et al. Type 2 diabetes among North American children and adolescents: an epidemiologic review and a public health perspective. J Pediatr. 2000;136:664-672.

9. Strauss RS, Pollack HA. Epidemic increase in childhood overweight, 1986-1998. JAMA. 2001;286:2845-2848.

10. Ogden $C L$, Flegal KM, Carroll MD, Johnson CL. Prevalence and trends in overweight among US children and adolescents, 19992000. JAMA. 2002;288:1728-1732.

11. Pinhas-Hamiel O, Dolan LM, Daniels SR, Standiford D, Khoury PR, Zeitler $\mathrm{P}$. Increased incidence of non-insulin-dependent diabetes mellitus among adolescents. J Pediatr. 1996;128:608-615.

12. Dabelea D, Hanson RL, Bennett PH, Roumain J, Knowler WC, Pettitt DJ. Increasing prevalence of Type II diabetes in American Indian children. Diabetologia. 1998;41:904-910.

13. National Center for Health Statistics. Analytic Guidelines: The Third National Health and Nutrition Examination and Survey, NHANES III (1988-1994). October, 1996. National Center for Health Statistics, Centers for Disease Control. Hyattsville, Maryland. Available at: http://www. cdc.gov/nchs/data/nhanes/nhanes3/cdrom/nchs/manuals/nh3guide.pdf.

14. Turner R, Stratton I, Horton V, et al, for the UK Prospective Diabetes Study (UKPDS) Group. UKPDS 25: autoantibodies to islet-cell cytoplasm and glutamic acid decarboxylase for prediction of insulin requirement in type 2 diabetes. Lancet. 1997;350:1288-1293.

15. World Health Organization. WHO expert committee on diabetes mellitus, 2nd report. Technical Report Series, No. 646. Geneva: World Health Organization; 1980.

16. Expert Committee on the Diagnosis and Classification of Diabetes Mellitus. Report of the expert committee on the diagnosis and classification of diabetes mellitus. Diabetes Care. 1997;20:1183-1197.

17. American Diabetes Association. Report of the expert committee on the diagnosis and classification of diabetes mellitus. Diabetes Care. 2003;26(Suppl 1):S5-S20.

18. U. S. Preventive Services Task Force. Screening for type 2 diabetes in adults: recommendations and rationale. Ann Int Med. 2003;138:212-214

19. Harris R, Donahue K, Rathore SS, Frame P, Woolf SH, Lohr KN. Screening adults for type 2 diabetes: a review of the evidence for the U.S. Preventive Services Task Force. Ann Int Med. 2003;138:215-229.

20. CDC Diabetes Cost-Effectiveness Study Group. The cost-effectiveness of screening for type 2 diabetes. JAMA. 1998;280:1757-1763.

21. Berger B, Stenstrom G, Sundkvist G. Random C peptide in the classification of diabetes. Scand J Clin Lab Invest. 2000;60:687-693.

22. Reider CR, Halter CA, Castelluccio PF, Oakes D, Nichols WC, Foroud T. Reliability of reported age at onset for Parkinson's disease. Mov Disord. 2003;18:275-279. 